$1685 a$ USE OF THE KURTOSIS STATISTIC TO BETTER ESTIMATE THE EFFECT OF NOISE AND SOLVENT PROFESSIONAL EXPOSURE ON HEARING THRESHOLD

${ }^{1} \mathrm{~A}$ Fuente, ${ }^{2} \mathrm{~W}$ Qiu, ${ }^{3} \mathrm{M}$ Zhang $\mathrm{M},{ }^{3} \mathrm{H}$ Xia, ${ }^{4} \mathrm{C}$ Kardous, ${ }^{5} \mathrm{P}$ Campo, ${ }^{4} \mathrm{TC}$ Morata. ${ }^{1} \mathrm{E}$ cole d'Orthophonie et d'Audiologie, Université de Montréal, Montréal, Canada; ${ }^{2}$ Auditory Research Laboratory, State University of New York, Plattsburgh, USA; 3 Institute of Environmental and Occupational Health, Zhejiang Provincial Centre for Disease Control and Prevention, China; ${ }^{4}$ National Institute for Occupational Safety and Health, Cincinnati, USA; 5 Institut National de Recherche et de Sécurité, Vandouvre Cedex, France

\subsection{6/oemed-2018-ICOHabstracts. 1475}

Introduction The aim of this exploratory study was to examine whether the kurtosis metric can contribute to investigations of the effects of combined exposure to noise and solvents on human hearing thresholds.

Methods Twenty factory workers exposed to noise and solvents along with 20 workers of similar age exposed only to noise in southern China were investigated using pure-tone audiometry (1000-8000 Hz). Exposure histories and shift-long noise recording files were obtained for each participant. The data was used in the calculation of their Cumulative Noise Exposure (CNE) which was adjusted using the kurtosis data recorded for each worker. Passive samplers were used to collect solvent concentrations for each worker exposed to solvents over the full work shift.

Results We observed an interaction between noise exposure and solvents for the hearing threshold at $6000 \mathrm{~Hz}$. This effect was observed only when the CNE level was adjusted by the kurtosis metric.

Disclaimer The views expressed in this publication are those of the authors and do not necessarily represent the views of the National Institute for Occupational Safety and Health.

\section{$1685 \mathrm{~b}$ HEARING PROCESS}

M Sánchez Fuentes. INSHT, Madrid, Spain

\subsection{6/oemed-2018-ICOHabstracts. 1476}

Introduction Many workers suffer from hearing dysfunctions, even if their exposure to noise and chemicals is within the established exposure limits. The mechanisms of action for combined exposure possible can be determined by sound perception indicators, and in this sense the appropriate health assessment can be performed.

Methods This exposure is based on the review of medical literature about the hearing process.

Result The hazardous synergistic effects of some chemicals and noise can be well understood with a deep knowledge of the hearing process. Pure-tone air-conduction audiometry is the most used method to check the state of the worker's auditory system, but it has been proven to be insufficient when hearing loss is due to a combined exposure to noise and ototoxic agents, since it does not allow the source of the problem to be correctly identified.

Discussion The hearing system can be affected not only by the noise, but also by its combination with ototoxic chemical agents, this damage can affect from the cochlea to the higher auditory pathways. It's important to use tools that evaluate the auditory system comprehensively, using tests that help to differentiate between the individual or the combined effects of noise and chemicals on hearing, such as the distortion product otoacoustic emissions. Furthermore, exposure limits to noise and ototoxic chemical agents lower than those set out in should be considered, taking into account their synergistic effect.

\section{C OTOACOUSTIC EMISSIONS ARE SENSITIVE TO OXIDATIVE STRESS IN HUMANS EXPOSED TO NOISE AND STYRENE}

${ }^{1} \mathrm{R}$ Sisto, ${ }^{1} \mathrm{~L}$ Cerini, ${ }^{1} \mathrm{~F}$ Sanjust, ${ }^{1} \mathrm{G}$ Tranfo, ${ }^{1} \mathrm{E}$ Paci, ${ }^{1} \mathrm{D}$ Pigini, ${ }^{2} \mathrm{~A}$ Moleti. ${ }^{1}$ INAIL Research, Department of Occupational and Environmental Medicine, Epidemiology and Hygiene, Monteporzio Catone, Italy; ${ }^{2}$ University of Rome Tor Vergata, Department of Physics, Rome, Italy

\subsection{6/oemed-2018-ICOHabstracts. 1477}

Introduction Evidence for synergistic interaction between noise and styrene in inducing cochlear damage can be found in the literature. At present a mechanistic explanation of such an interaction is not available, although animal studies are shedding light on the phenomenon. At the aim of clarifying the effect of simultaneous exposure to noise (mild level) and styrene on the cochlear functionality, distortion product otoacoustic emissions (DPOAEs) were used as biomarkers of the cochlear status. In this work, the correlation between the DPOAE level and blood concentration of oxidative stress biomarkers is studied in a sample of subjects professionally exposed to styrene. This study is aimed at investigating the role of the oxidative stress in the inner ear damage in workers exposed to styrene.

Methods Nine subjects exposed to styrene in a fibreglass factory and eight control subjects were enrolled in this study. The mean age was the same in the exposed and control groups. The airborne concentration of styrene was evaluated by means of ambiental and personal samplers. The end shift urinary concentration of the phenylglyoxylic (PGA) and mandelic acid (MA) was also evaluated. Distortion product otoacoustic emissions were measured in the exposed workers and in a control group. The DPOAE component generated by a nonlinear mechanism, characterised by zero latency, and the long-latency component, generated by a linear reflection mechanism, were separately analysed, using a time-frequency domain wavelet filter for component unmixing.

The urinary concentration of the DNA and RNA oxidation products, namely 8-oxo-7,8-dihydroguanine (oxoGua), 8-oxo7,8-dihydro-20-deoxyguanosine (oxodGuo), and 8-oxo-7,8dihydroguanosine (oxoGuo), were evaluated and correlated to the DPOAE level.

Results A statistically significant negative correlation was found between the DPOAE level and the PGA and MA urinary concentration. A dose response relation was proposed correlating the styrene concentration, the exposure duration and the DPOAE level, used as outcome variables.

High levels of the oxidative damage biomarkers were found in the workers exposed to high levels of styrene. Significant negative correlation was found between the otoacoustic emission distortion component levels and the concentration of the oxoGuo biomarker. 